\title{
CATEGORY WEIGHT: NEW IDEAS CONCERNING LUSTERNIK-SCHNIRELMANN CATEGORY
}

\author{
YULI B. RUDYAK \\ Mathematisches Institut, Universität Heidelberg \\ Im Neuenheimer Feld 288, D-69120 Heidelberg 1, Germany \\ E-mail: july@mathi.uni-heidelberg.de
}

Introduction. The concept of category weight was introduced by Fadell-Husseini $[\mathrm{FH}]$ and developed by Rudyak and Strom. Here we give a survey, some further development and applications of category weight.

The Lusternik-Schnirelmann category of a topological space $X$, cat $X$, is defined as the minimal number $k$ such that $X$ admits a numerable covering $\left\{A_{1}, \ldots, A_{k+1}\right\}$ where each $A_{i}$ is contractible in $X$. Lusternik and Schnirelmann [LS] introduced the invariant cat $X$ for manifolds. They proved that, for every connected smooth $\left(=C^{\infty}\right)$ closed manifold $M$,

$$
1+\operatorname{cat} M \leq \text { Crit } M:=\min \left\{\operatorname{crit} f \mid f \in C^{\infty}(M, R)\right\}
$$

where crit $f$ is the number of critical points of a smooth real-valued function $f$ on $M$. Afterwards Fox [Fox] suggested considering cat $X$ as an invariant of a space $X$. The basic information concerning the Lusternik-Schnirelmann category can be found in [Fox], $[\mathrm{Sv}],[\mathrm{J}]$.

This (homotopy) invariant is quite far from other invariants like homotopy and homology groups, so it is difficult to compute cat $X$. For example, in $[\mathrm{G}]$, Ganea asked whether $\operatorname{cat}\left(X \times S^{n}\right)=\operatorname{cat} X+1, n>0, X$ connected. The affirmative claim is usually referred to as the Ganea conjecture. Recently it was disproved by Iwase [I], but it is still unclear whether it is true for manifolds. Here is another rather naive question. Let $f: M \rightarrow N$ be a map of degree 1 of closed manifolds. Is it true that cat $M \geq$ cat $N$ ?

One of the favorite and famous ways to estimate the Lusternik-Schnirelmann category is a so-called cup-length estimation (Froloff-Elsholz [FE], Eilenberg). Namely, if $u_{1} \cdots u_{n} \neq 0$ for some $u_{i} \in \widetilde{H}^{*}(X)$ then cat $X \geq n$. The idea of the proof is quite simple: if $X=A_{1} \cup \cdots \cup A_{n}$ where each $A_{i}$ is contractible in $X$ then $u_{i} \mid A_{i}=0$, and so $u_{1} \cdots u_{n} \mid\left(A_{1} \cup \cdots \cup A_{n}\right)=0$. However, the cup-length estimation is not perfect. For example, if $L=L_{p}^{2 n+1}$ is the lens space with $p$ an odd prime, then cat $L=2 n+1$ (Krasnosel'ski,

1991 Mathematics Subject Classification: Primary 55M30; Secondary 55N20, 55S30, 58F05.

The paper is in final form and no version of it will be published elsewhere. 
1955), while the cup-length estimation gives us cat $L \geq n+1$ only. Fadell-Husseini refined the cup-length estimation by suggesting that elements $u_{i}$ be equipped with "weights". Speaking informally, we say that the category weight of $u(\operatorname{cwgt} u)$ is $\geq k$ if

$$
u \mid\left(A_{i_{1}} \cup \cdots \cup A_{i_{k}}\right)=0
$$

for $A_{i}$ as above. Clearly, cat $X \geq \operatorname{cwgt} u$ if $u \neq 0$. Furthermore, $\operatorname{coggt}\left(u_{1} \cdots u_{n}\right) \geq$ $\sum$ cwgt $u_{i}$, and, since cwgt $u_{i} \geq 1$, we conclude that

$$
\text { cat } X \geq \operatorname{cwgt}\left(u_{1} \cdots u_{n}\right) \geq \sum \operatorname{cwgt} u_{i} \geq n
$$

provided $u_{1} \cdots u_{n} \neq 0$. Certainly, this improves the cup-length estimation. For example, this establishes a short calculation of cat $L_{p}^{2 n+1}$, see $[\mathrm{FH}]$. So, it seems reasonable to find (indecomposable) elements of high category weight and, more generally, to be able to compute category weight. Unfortunately, category weight is not a homotopy invariant, i.e., cwgt $h^{*} u$ is not necessarily equal to cwgt $u$ for a homotopy equivalence $h$. This makes category weight difficult for calculations.

Because of this, it makes sense to introduce a homotopy invariant version of category weight as the author did in a talk at the AMS Summer Research Institute, Seattle, July 1996 (see [R2]). This invariant is called strict category weight and denoted by swgt. A similar concept was also introduced by Strom [S2]. There are many ways to define swgt $u, u \in H^{*}(X)$. One of them is:

swgt $u=\min \left\{\operatorname{cwgt} h^{*} u\right\}$ where $h$ runs over all homotopy equivalences $Y \rightarrow X$.

Clearly, cwgt $u \geq \operatorname{swgt} u$, so we can use swgt to estimate cwgt. Furthermore, swgt has better multiplicative properties than cwgt, see $\S 3$. It turns out that swgt is quite manageable: for example, it is possible both to find many elements of high swgt (see §4) and to apply swgt to certain geometric problems (see $\S \S 6,7)$. Also, notice that strict category weight yields a decreasing filtration $\left\{F_{n}(X)\right\}$ of $H^{*}(X), F_{n}(X):=\left\{u \in H^{*}(X) \mid\right.$ swgt $\left.u \geq n\right\}$.

Here is another description of swgt: we have $X \simeq B \Omega X$, the classifying space for the loop space of $X$. Let $\left\{B_{n} \Omega X\right\}$ be the Milnor filtration of $B \Omega X$, and let $i_{n}: B_{n} \Omega X \subset$ $B \Omega X \simeq X$. (Notice that $i_{n}$ is homotopy equivalent to a map (1.5) described in $\S 1$.) Then

$$
\operatorname{swgt} u=\sup \left\{k \mid i_{k}^{*}(u)=0\right\} .
$$

In other words, the above filtration $\left\{F_{n}(X)\right\}$ is just the filtration

$$
\operatorname{Ker}\left\{i_{n}^{*}: H^{*}(X) \rightarrow H^{*}\left(B_{n} \Omega X\right)\right\} .
$$

This also shows how to apply the Eilenberg-Moore spectral sequence to the study of the Lusternik-Schnirelmann category, cf. [To].

It is clear that category weight can be defined in more general situations. For example, we can consider an arbitrary (extraordinary) cohomology theory instead of $H^{*}(-)$. In this paper we consider even a little bit more general functors then cohomology.

Throughout this paper, we reserve the term "map" for continuous functions and the term "inessential map" for homotopy trivial maps (i.e., for maps which are homotopic to constant maps).

We use the sign $\simeq$ for homotopy of maps, and we write $f \simeq^{\bullet} g$ when there is a pointed homotopy between pointed maps $f$ and $g$. 
The reduced cone of a pointed map $f$ is denoted by $C f$.

"Connected" always means "path connected".

Given a pointed topological space $X$, the (reduced) cohomology group $H^{k}(X ; \pi)$ is defined as $[X, K(\pi, n)]$ (pointed homotopy classes) where the Eilenberg-Mac Lane space $K(\pi, n)$ is assumed to be ( homotopy equivalent to) a $C W$-space. Two exceptions to this agreement (in 3.10 and 4.2) are mentioned explicitly.

Given $u \in H^{k}(X)$, the notation $\left.u\right|_{\pi_{k}(X)}=0$ means that $\langle u, a\rangle=0$ for every $a \in$ $\operatorname{Im}\left\{h: \pi_{k}(X) \rightarrow H_{k}(X)\right\}$ where $h$ is the Hurewicz homomorphism.

The paper is organized as follows. In $\S 1$ we consider the Lusternik-Schnirelmann category. In $\S 2$ we give a definition and background properties of (strict) category weight. In $\S 3$ we demonstrate multiplicative properties of (strict) category weight. In $\S 4$ we explain how to find elements of high category weight. In $\S 5$ we show how to use swgt for control of the Lusternik-Schnirelmann category and in $\S 6$ we apply this technique to manifolds. In particular, we prove special cases of the Ganea conjecture and the degree conjecture. In $\S 7$ we apply our technique to the famous Arnold conjecture about symplectic fixed points. In fact, we idicate how to prove it for closed symplectic manifolds $(M, \omega)$ with $\left.\omega\right|_{\pi_{2}(M)}=0=\left.c_{1}\right|_{\pi_{2}(M)}$.

\section{Lusternik-Schnirelmann category}

1.1. Definition. (a) ([Fox], [Fet], [BG]) Given a map $\varphi: A \rightarrow X$, we say that cat $\varphi \leq k$ if there is a numerable covering $U_{1}, \ldots, U_{k+1}$ of $A$ such that $\varphi \mid U_{i}$ is inessential for every $i$. Then cat $\varphi=k$ if $k$ is minimal with this property. Also, we set $\operatorname{cat} \varphi=-1$ if $A=\emptyset$.

(b) If $i: A \rightarrow X$ is an inclusion then we set $\operatorname{cat}_{X} A:=\inf \{$ cat $i\}$ where $i$ runs over all the inclusions $i: U \rightarrow X$ of neighborhoods $U$ of $A$.

(c) ([LS]) We define the Lusternik-Schnirelmann category cat $X$ of a space $X$ by setting cat $X:=\operatorname{cat} 1_{X}=\operatorname{cat}_{X} X$.

1.2. Lemma, Let $f: X \rightarrow Y$ be a map with $Y$ connected, and let $x_{0} \in X$ be an arbitrary point. If cat $f=k$ then there is a numerable covering $\left\{B_{1}, \ldots, B_{k+1}\right\}$ such that $f \mid B_{i}$ is inessential and $x_{0} \in B$ for every $i$.

P r o of. Let $\left\{A_{1}, \ldots, A_{k+1}\right\}$ be a numerable covering of $X$ with $f \mid A_{i}$ inessential, and let $\left\{\varphi_{1}, \ldots, \varphi_{k+1}\right\}$ be a partition of unity dominated by $\left\{A_{1}, \ldots, A_{k+1}\right\}$. We set

$$
C_{i}:=\varphi_{i}^{-1}[1 /(2 k+2), 1]
$$

and $B_{i}:=C_{i} \cup\left\{x_{0}\right\}$. It is easy to see that $\left\{B_{i}\right\}$ is the desired covering.

1.3. Proposition ([BG]). (i) For every diagram $A \stackrel{\varphi}{\rightarrow} Y \stackrel{f}{\rightarrow} X$ we have cat $f \varphi \leq$ $\min \{\operatorname{cat} \varphi$, cat $f\}$. In particular, cat $f \leq \min \{\operatorname{cat} X$, cat $Y\}$.

(ii) If $\varphi \simeq \psi: A \rightarrow X$ then $\operatorname{cat} \varphi=\operatorname{cat} \psi$.

(iii) If $h: Y \rightarrow X$ is a homotopy equivalence then cat $\varphi=\operatorname{cat} h \varphi$ for every $\varphi: A \rightarrow$ $X$.

Notice that, in view of 1.3 (iii), cat $X$ is a homotopy invariant of $X$. Also, it is easy to see that cat $X \leq \operatorname{dim} X$ for every connected $C W$-space $X$. 
1.4. Proposition ([B], [Fox]). For any two connected $C W$-spaces $X, Y$ we have

$$
\max \{\operatorname{cat} X, \operatorname{cat} Y\} \leq \operatorname{cat}(X \times Y) \leq \operatorname{cat} X+\operatorname{cat} Y .
$$

Let $X$ be a connected space. Take a point $x_{0} \in X$, set

$$
P X=P\left(X, x_{0}\right)=\left\{\omega \in X^{I} \mid \omega(0)=x_{0}\right\}
$$

and consider the fibration $p: P X \rightarrow X, p(\omega)=\omega(1)$ with the fiber $\Omega X$. Given a natural number $k$, we use the short notation

$$
p_{k}: P_{k}(X) \rightarrow X
$$

for the map

$$
\underbrace{p_{X} *_{X} \cdots *_{X} p_{X}}_{k \text { times }}: \underbrace{P X *_{X} \cdots *_{X} P X}_{k \text { times }} \rightarrow X
$$

where $*_{X}$ denotes the fiberwise join over $X$, see e.g. $[\mathrm{J}]$. In particular, $P_{1}(X)=P X$.

1.6. Theorem ([Sv, Theorems 3 and 19']). Let $\varphi: A \rightarrow X$ be a map with $X$ connected. Then cat $\varphi<k$ iff there is a map $\psi: A \rightarrow P_{k}(X)$ such that $p_{k} \psi=\varphi$.

For future references we fix the following simple information, the proofs can be found e.g. in $[\mathrm{R} 2]$.

1.7. Proposition. (i) $P_{k}(X)$ is a fibration over $X$;

(ii) If $X$ has the homotopy type of a $C W$-space then $P_{-k}(X)$ does;

(iii) cat $P_{k}(X) \leq k-1$.

1.8. Proposition ([Sv, Th. 21]). The map $p_{2}: P_{2}(X) \rightarrow X$ is homotopy equivalent over $X$ to the map $\varepsilon: S \Omega X \rightarrow X$.

The concept of Lusternik-Schnirelmann category can be generalized as follows. Let $\mathscr{T}$ be the category of pointed spaces and pointed maps. Let $\mathscr{E}$ be the category whose objects are pairs (set, subset) and whose morphisms $(U, V) \rightarrow\left(U^{\prime}, V^{\prime}\right)$ are functions $f: U \rightarrow U^{\prime}$ with $f(V) \subset V^{\prime}$. Given a contravariant functor $F: \mathscr{T} \rightarrow \mathscr{E}$ with $F(X)=(U, V)$ for some $X \in \mathscr{T}$, the notation $u \in F(X)$ means that $u \in U$. We say that $u \in F(X)=(U, V)$ is trivial if $u \in V$. Given $u \in F(Y)$ and $f: X \rightarrow Y$, we write $f^{*} u$ for $F(f)(u)$. Furthermore, given $A \subset X$, we denote $i^{*} u$ by $u \mid A$.

1.9. Definition. Let $F: \mathscr{T} \rightarrow \mathscr{E}$ be a contravariant functor satisfying the following properties:

(1) (weak homotopy property) If $f \simeq^{\bullet} g: X \rightarrow Y$ then $f^{*} u$ is trivial if $g^{*} u$ is.

(2) (triviality property) $F(\mathrm{pt})$ has the form $(U, U)$, i.e., every $u \in F(\mathrm{pt})$ is trivial.

Let $X$ be a connected pointed space. Given $u \in F(X)$, we define the Lusternik-Schnirelmann category of $u$, cat $u$, to be the minimal $k$ with the following property: there is a numerable covering $\left\{A_{1}, \ldots, A_{k+1}\right\}$ where each $A_{i}$ is a pointed subspace of $X$ and $u \mid A_{i}$ is trivial for every $i=1, \ldots, k$.

1.10. Examples. (a) Given a pointed topological space $Y$, set $F(X)=T_{Y}(X):=$ $(U, V)$ where $U$ is the set of all pointed maps $X \rightarrow Y$ and $V$ is the subset of all inessential 
(under non-pointed homotopy) maps. (The action of $F$ on morphisms is given by the composition.) Then, the definition above leads to the invariant cat $f$ defined in 1.1.

(b) Every pointed set $\left(U, u_{0}\right)$ can be considered as the pair $\left(U,\left\{u_{0}\right\}\right)$ of sets. Thus, every functor from $\mathscr{T}$ to the category of pointed sets can be regarded as a functor $\mathscr{T} \rightarrow \mathscr{E}$.

(c) Every abelian group can be regarded as a pointed set (with base point 0). So, every functor from $\mathscr{T}$ to the category of abelian groups can be treated as a functor $\mathscr{T} \rightarrow \mathscr{E}$.

(d) (Fary $[\mathrm{F}]$ ) Because of (c), a reduced cohomology theory (not necessarily additive) $E^{*}(-)$ on $\mathscr{T}$ yields the functor $X \mapsto F(X):=\left(E^{i}(X),\{0\}\right)$. Then, given $u \in E^{i}(X)$, we have the invariant cat $u$, the Lusternik-Schnirelmann category of the class $u$.

(e) Given $X \in \mathscr{T}$, set $F(X)=(U, V)$ where $U$ is the set of fiberwise homotopy equivalence classes of fibrations over $X$ and $V$ consists of fibrations which admit a section. The action of $F$ on morphisms is given by passing to induced fibrations. If $u \in F(X)$ is the equivalence class of a fibration $\xi$, then cat $u$ is the genus of $\xi$, [Sv]; another name is the sectional category, secat $\xi,[\mathrm{J}]$.

Notice that the example (e) generalizes example (a) since cat $f=$ secat $f^{*} \xi$ where $\xi=\left\{p_{1}: P Y \rightarrow Y\right\}$ for $f: X \rightarrow Y$.

1.11. Proposition. Let $X$ be a connected pointed space.

(i) cat $f^{*} u \leq \min \{$ cat $f$, cat $u\}$ for every $f: X \rightarrow Y$ in $\mathscr{T}$ and every $u \in F(Y)$.

(ii) If $f \simeq^{\bullet} g: X \rightarrow Y$ then cat $f^{*} u=$ cat $g^{*} u$ for every $u \in F(Y)$.

(iii) If $h: X \rightarrow Y$ is a pointed homotopy equivalence then cat $h^{*} u=$ cat $u$ for every $u \in F(Y)$.

\section{Category weight}

2.1. Definition. Let $F: \mathscr{T} \rightarrow \mathscr{E}$ be a functor as in 1.9 , and let $u \in F(X)$. We do not require $X$ to be connected.

(a) We define the category weight of $u$ (denoted by cwgt $u$ ) by setting

$$
\operatorname{cwgt} u=\sup \{k|u| A \text { is trivial whenever cat } X<k\}
$$

where $A$ runs over all pointed closed subsets of $X$

(b) We define the strict category weight of $u$ (denoted by swgt $u$ ) by setting

$\operatorname{swgt} u=\sup \left\{k \mid \varphi^{*} u\right.$ is trivial for every map $\varphi: A \rightarrow X$ in $\mathscr{T}$ with $\left.\operatorname{cat} \varphi<k\right\}$.

Notice that cwgt $u=\infty=\operatorname{swgt} u$ for every trivial element $u$.

In particular, the (strict) category weight of a map (cf. 1.10(a)) and a cohomology class (cf.1.10(d)) is defined. Category weight was defined by Fadell and Husseini [FH] (for $\left.F=H^{*}(-)\right)$. Strict category weight was defined by Rudyak for $F=E^{*}(-)$ as in $1.10(\mathrm{~d})$ and Strom for $F$ as in 1.10(a), cf. [R2], [S2]; Strom calls it essential category weight. I must also note that Strom prefers to say that the (strict) category weight of a trivial element is not defined.

2.2. TheOREM (cf. [R2]). (i) $\operatorname{swgt} u \leq \operatorname{cwgt} u$, and cwgt $u \leq$ cat $X$ provided $u$ is not a trivial element. Furthermore, for every map $f: Y \rightarrow X$ in $\mathscr{T}$ we have cat $f \geq \operatorname{swgt} u$ provided $f^{*} u$ is non-trivial. Finally, swgt $u \geq 1$ for every $u$.

(ii) For every inclusion $i: A \rightarrow X$ in $\mathscr{T}$ we have cwgt $i^{*} u \geq \operatorname{cwgt} u$. 
(iii) For every map $f: Y \rightarrow X$ in $\mathscr{T}$ we have swgt $f^{*} u \geq$ swgt $u$.

(iv) If $f \simeq^{\bullet} g: X \rightarrow Y$ then $\operatorname{cwgt} f^{*} u=\operatorname{cwgt} g^{*} u$ and swgt $f^{*} u=\operatorname{swgt} g^{*} u$.

(v) swgt $u=\operatorname{swgt} h^{*} u$ for every pointed homotopy equivalence $h$.

Notice that properties (iii) and (v) of 2.2 are not valid for cwgt even if $f=H^{*}(-)$, see [R2], [S2]. In other words, category weight is not a homotopy invariant, and this is the main motivation for introducing strict category weight. The following proposition gives us another description of strict category weight.

2.3. Proposition. Let $X$ be a connected pointed space. Then for every $u \in F(X)$ we have:

(i) $\operatorname{swgt} u=\sup \left\{k \mid p_{k}^{*}(u)\right.$ is trivial $\}$;

(ii) If swgt $u=k$ then $\operatorname{cwgt} p_{k+1}^{*} u=k$;

(iii) swgt $u=\min _{f \in \mathscr{F}}\left\{\operatorname{cwgt} f^{*} u\right\}$ where $\mathscr{F}$ is the class of all maps $f: Y \rightarrow X$ in $\mathscr{T}$.

Proof. (i) See $[R 2,1.8(v)]$.

(ii) Since swgt $u=k$, we conclude that, by (i), $p_{k+1}^{*} u$ is non-trivial. Now

$$
k=\operatorname{swgt} u \leq \operatorname{swgt} p_{k+1}^{*} u \leq \operatorname{cwgt} p_{k+1}^{*} u \leq \operatorname{cat} P_{k+1} X \leq k .
$$

(iii) Clearly, swgt $u \leq \operatorname{swgt} f^{*} u \leq \operatorname{cwgt} f^{*} u$. Now, if swgt $u=k$ then, by (ii) swgt $u=$ $\operatorname{cwgt} p_{k+1}^{*} u$.

2.4. TheOrem. Let $X$ be a connected pointed space.

(i) For every $f: X \rightarrow Y$ in $\mathscr{T}$ and every $u \in F(Y)$ we have

$$
\text { cat } f \geq(\operatorname{swgt} u) \operatorname{cat}\left(f^{*} u\right) \text {. }
$$

In particular, for every diagram $X \stackrel{f}{\rightarrow} Y \stackrel{g}{\rightarrow} Z$ we have cat $f \geq(\operatorname{swgt} g) \operatorname{cat}(g f)$.

(ii) For every $u \in F(X)$ we have

$$
\operatorname{cat}_{X} A \geq(\operatorname{cwgt} u) \operatorname{cat}(u \mid A) \text {. }
$$

In particular, for every map $f: X \rightarrow Y$ we have $\operatorname{cat}_{X} A \geq(\operatorname{cwgt} f) \operatorname{cat}(f \mid A)$.

Formula (ii) was found by Strom [S2], formula (i) is an obvious analog of (ii). This theorem improves properties 1.11(i) and 2.2(i). Notice that the proof does not use properties (1) and (2) of 1.9.

Proof. We prove only (i). Let cat $f=n$, swgt $u=k>0$. We must prove that $\operatorname{cat}\left(f^{*} u\right) \leq p:=[n / k]$. Let $\left\{A_{1}, \ldots, A_{n+1}\right\}$ be a numerable covering of $X$ such that $f \mid A_{i}$ is inessential for every $i$. Without loss of generality we can assume $Y$ to be connected, and so, by 1.2 , we can assume that each $A_{i}$ contains the base point. We set

$$
\begin{gathered}
B_{1}:=A_{1} \cup \cdots \cup A_{k}, B_{2}=A_{k+1} \cup \cdots \cup A_{2 k}, \ldots, \\
B_{p}=A_{(p-1) k+1} \cup \cdots \cup A_{p k}, B_{p+1}=A_{p k+1} \cup \cdots \cup A_{n+1} .
\end{gathered}
$$

Since $\operatorname{cat}\left(f \mid B_{j}\right)<k$, we conclude that, by $2.2(\mathrm{i}),\left(f \mid B_{j}\right)^{*} u$ is trivial. So, $\left(f^{*} u\right) \mid B_{j}$ is trivial for every $j=1, \ldots, p+1$. Thus, cat $f^{*} u \leq p$. 
2.5. Corollary ([S2]). For every $f: X \rightarrow Y$ in $\mathscr{T}$ with $X$ connected and every $u \in F(Y)$ we have

$$
\text { cwgt } f^{*} u \geq(\operatorname{cwgt} f) \operatorname{swgt} u, \operatorname{swgt} f^{*} u \geq(\operatorname{swgt} f) \operatorname{swgt} u \text {. }
$$

2.6. Proposition ([R2], [S2]). Let $X$ be a $C W$-space, and let $f: X \rightarrow Y, X \in \mathscr{T}$ be an essential map such that $\pi_{i}(Y)=0$ for $i>m$. Then cwgt $f \leq m$. In particular, if $E$ is a spectrum with $\pi_{i}(E)=0$ for $i>m$ then cwgt $u \leq q+m$ for every $u \in E^{q}(X), u \neq 0$.

3. Multiplicative properties. Let $F$ be a functor as in 1.9. Given a Puppe sequence $X \stackrel{f}{\rightarrow} Y \rightarrow C f$ in $\mathscr{T}$, we conclude, by 1.9(1), that the image of the composition

$$
F(C f) \rightarrow F(Y) \rightarrow F(X)
$$

consists of trivial elements.

3.1. Definition. A functor $F$ as 1.9 is called half-exact if, for every pointed polyhedral pair $(X, A)$, the sequence

$$
F(X \cup C A) \stackrel{j^{*}}{\rightarrow} F(X) \stackrel{i^{*}}{\rightarrow} F(A)
$$

is "exact", i.e., $i^{*} u$ is trivial iff $u=j^{*} v$ for some $v \in F(X \cup C A)$. Here $i: A \rightarrow X$ and $j: X \rightarrow X \cup C A$ are the inclusions. Given $\left(U_{1}, V_{1}\right), \ldots,\left(U_{n}, V_{n}\right) \in \mathscr{E}$, we set

$$
\left(U_{1}, V_{1}\right) \times \cdots \times\left(U_{n}, V_{n}\right):=\left(U_{1} \times \cdots U_{n}, V_{1} \times \cdots V_{n}\right) .
$$

Let $E, F$ be two half-exact functors, and let $G$ be an arbitrary functor as in 1.9. Suppose that, for every two polyhedra $X, Y$ (not necessarily finite), there is a natural transformation (where $\times$ is as described in $(3.2)$ )

$$
m: E(X) \times F(Y) \rightarrow G(X \wedge Y) .
$$

This transformation yields a transformation

$$
\tau: E(X) \times F(Y) \stackrel{m}{\rightarrow} G(X \wedge Y) \stackrel{\lambda}{\rightarrow} G(X \times Y),
$$

where $\lambda$ shrinks the wedge $X \vee Y$. We set $u \bigotimes v:=\tau(u, v)$ for $u \in E(X), v \in F(Y)$. If $X=Y$ and $\Delta: X \rightarrow X \times X$ is the diagonal, we set $u \bullet v:=\Delta^{*}(u \bigotimes v) \in G(X)$.

3.4. TheOREM (cf. [FH], [R2]). (i) For every pointed connected polyhedron $X$ and every $u \in E(X), v \in F(X)$ we have

$$
\operatorname{cwgt}(u \bullet v) \geq \operatorname{cwgt} u+\operatorname{cwgt} v .
$$

(ii) For every pointed connected $C W$-space $X$ and every $u \in E(X), v \in F(X)$ we have

$$
\operatorname{swgt}(u \bullet v) \geq \operatorname{swgt} u+\operatorname{swgt} v .
$$

Proof. (i) First, assume that cwgt $u=k<\infty$, cwgt $v=l<\infty$. Take a pointed closed subspace $A$ of $X$ with cat $X<k+l$. Then, clearly, $A \subset \bigcup_{i=1}^{k+l} V_{i}$ where each $V_{i}$ is open and contractible in $X$. Since $A$ is closed, there is a subdivision of $X$ with the following property: every simplex $e$ with $e \cap A \neq \emptyset$ is contained in some $V_{i}$, cf. [W, Theorem 35]. We let $A_{i}, i=1, \ldots, r_{n}$ be the union of all simplexes contained in $V_{i}$. 
Then $A \subset A_{1} \cup \cdots \cup A_{k+l}$ where each subpolyhedron $A_{i}$ is contractible in $X$. We set $K:=A_{1} \cup \cdots \cup A_{k} \cup\{*\}, L:=A_{k+1} \cup \cdots \cup A_{k+l} \cup\{*\}$ and let

$$
i_{K}: K \subset X, i_{L}: L \subset X, j_{K}: X \subset X \cup C K, j_{L}: X \subset X \cup C L, h: X \subset X \cup C(K \cup L)
$$

be the inclusions. Consider the commutative diagram

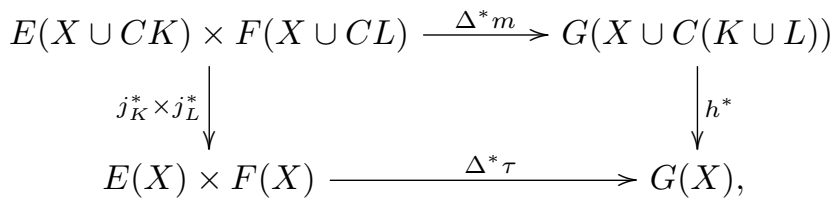

where, for the top row, we have used the canonical homotopy equivalence

$$
\left(X \cup C X^{\prime}\right) \wedge\left(Y \cup C Y^{\prime}\right) \simeq X \times Y \cup C\left(\left(X \times Y^{\prime}\right) \cup\left(X^{\prime} \times Y\right)\right)
$$

which holds for any polyhedral pairs $\left(X, X^{\prime}\right)$ and $\left(Y, Y^{\prime}\right)$. Since both $i_{K}^{*} u$ and $i_{L}^{*} v$ are trivial, $u=j_{K}^{*} u^{\prime}, v=j_{L}^{*} v^{\prime}$ for some $u^{\prime} \in E(X \cup C K), v^{\prime} \in F(X \cup C L)$. Clearly, $u \bullet v=$ $h\left(u^{\prime} \bullet v^{\prime}\right)$. Thus, $(u \bullet v) \mid A$ is trivial since $A \subset K \cup L$.

If, say, cwgt $u=\infty$ then we must prove that $u \bullet v \mid A$ is trivial if cat $_{X} A<\infty$. Arguing as above, we conclude that there is a pointed subpolyhedron $K \subset X$ with $A \subset K$ and cat $_{X} K<\infty$. Then $u \mid K$ is trivial, and hence $(u \bullet v) \mid K$ is (take $L=*$ in the above diagram).

(ii) Let $\operatorname{swgt}(u \bullet v)=k$. Then, by $2.3\left(\right.$ ii), $\operatorname{cwgt} p_{k+1}^{*}(u \bullet v)=k$. Recall that every $C W$ space is homotopy equivalent to a polyhedron, and so, by 1.7(ii), there is a homotopy equivalence $\varepsilon: Y \rightarrow P_{k+1}(X)$ such that $Y$ is a polyhedron. We put $f=p_{k+1} \varepsilon: Y \rightarrow X$. Then $f^{*}(u \bullet v) \neq 0$, and

$$
k=\operatorname{swgt}(u \bullet v) \leq \operatorname{cwgt} f^{*}(u \bullet v) \leq \operatorname{cat} Y \leq k .
$$

Now

$$
\begin{aligned}
\operatorname{swgt}(u \bullet v) & =\operatorname{cwgt} f^{*}(u \bullet v) \geq \operatorname{cwgt} f^{*} u+\operatorname{cwgt} f^{*} v \geq \operatorname{swgt} f^{*} u+\operatorname{swgt} f^{*} v \\
& \geq \operatorname{swgt} u+\operatorname{swgt} v .
\end{aligned}
$$

3.5. TheOREM $([\mathrm{R} 2,1.14]) . \operatorname{swgt}(u \bigotimes v) \geq \operatorname{swgt} u+\operatorname{swgt} v$.

Notice that the corresponding inequality for cwgt is wrong, cf. 2.2(iii,v).

Certainly, we can consider not only two functors $E, F$ but any finite number of functors equipped with a natural transformation like (3.3).

3.6. ExAmples. (a) Given $n$ arbitrary spectra $E(1), \ldots, E(n)$ and $n$ pointed $C W$ spaces $X_{1}, \ldots, X_{n}$, we have the homomorphism

$$
\nu: E(1)^{*}\left(X_{1}\right) \otimes \cdots \otimes E(n)^{*}\left(X_{n}\right) \rightarrow(E(1) \wedge \cdots \wedge E(n))^{*}\left(X_{1} \times \cdots \times X_{n}\right),
$$

see $[\mathrm{Sw}]$. Furthermore, consider the correspondence (not a homomorphism!)

$$
\begin{gathered}
r: E(1)^{*}\left(X_{1}\right) \times \cdots \times E(n)^{*}\left(X_{n}\right) \rightarrow E(1)^{*}\left(X_{1}\right) \otimes \cdots \otimes E(n)^{*}\left(X_{n}\right), \\
r\left(u_{1}, \ldots, u_{n}\right)=u_{1} \otimes \cdots \otimes u_{n} .
\end{gathered}
$$

So, we have a natural transformation

$$
\tau:=\nu r: E(1)^{*}\left(X_{1}\right) \times \cdots \times E(n)^{*}\left(X_{n}\right) \rightarrow(E(1) \wedge \cdots \wedge E(n))^{*}\left(X_{1} \times \cdots \times X_{n}\right) .
$$


Now, by 3.5

$$
\operatorname{swgt}\left(u_{1} \otimes \cdots \otimes u_{n}\right) \geq \sum \operatorname{swgt} u_{i}
$$

for every $u_{i} \in E(i)^{*}\left(X_{i}\right)$. Furthermore, given $u_{i} \in E(i)^{*}(X)$, we have the element $u_{1} \bullet \cdots \bullet u_{n} \in(E(1) \wedge \cdots \wedge E(n))^{*}(X)$, and

$$
\operatorname{cwgt}\left(u_{1} \bullet \cdots \bullet u_{n}\right) \geq \sum \operatorname{cwgt} u_{i}, \quad \operatorname{swgt}\left(u_{1} \bullet \cdots \bullet u_{n}\right) \geq \sum \operatorname{swgt} u_{i}
$$

(in the first inequality $X$ is assumed to be a polyhedron).

(b) Given a ring spectrum $E$ with a multiplication $u: E \wedge E \rightarrow E$, for every $n$ we have the iterated multiplication

$$
u_{n}: \underbrace{E \wedge \cdots \wedge E}_{n \text { times }} \rightarrow E
$$

which yields a function

$$
\tau: E^{*}\left(X_{1}\right) \times \cdots \times E^{*}\left(X_{n}\right) \rightarrow(E \wedge \cdots \wedge E)^{*}\left(X_{1} \times \cdots \times X_{n}\right) \stackrel{\left(u_{n}\right)_{*}}{\rightarrow} E^{*}\left(X_{1} \times \cdots \times X_{n}\right)
$$

where the first arrow is $\tau$ from (a). In this case the element $u_{1} \bullet \cdots \bullet u_{n} \in E^{*}(X)$ is usually denoted by $u_{1} \cdots u_{n}$, and we have

$$
\text { cat } X \geq \operatorname{cwgt}\left(u_{1} \cdots u_{n}\right) \geq \sum \operatorname{cwgt} u_{i} \geq n
$$

if $u_{1} \cdots u_{n} \neq 0$. Certainly, this refines the cup-length estimation cat $X \geq n$.

3.8. EXAmple $([\mathrm{RO}])$. A closed connected symplectic manifold is a pair $\left(M^{2 n}, \omega\right)$ where $M$ is a connected closed smooth $2 n$-dimensional manifold and $\omega$ is a closed nondegenerate 2 -form. Notice that in this case $\omega^{n}$ is a volume form for $M$. In particular, $\omega$ yields a non-trivial de Rham cohomology class which we denote also by $\omega$. Since $\omega^{n} \neq 0$, we conclude that cat $M \geq n$. Rudyak-Oprea [RO] proved that swgt $\omega=2$ provided $\left.\omega\right|_{\pi_{2}(M)}=0$. Thus, if $\left(M^{2 n}, \omega\right)$ is a symplectic manifold with $\left.\omega\right|_{\pi_{2}(M)}=0$ then, by (3.7),

$$
\operatorname{cat} M \geq \operatorname{swgt} \omega^{n} \geq n \operatorname{swgt} \omega=2 n,
$$

and hence cat $M=2 n$ (because cat $M \leq \operatorname{dim} M$ ).

3.9. Examples. (a) ([S2]) Given two pointed spaces $A, B$, set $E(-)=T_{A}(-), F(-)=$ $T_{B}(-)$ and $G(-)=T_{A \wedge B}(-)$ (see 1.10(a)). We define $m: E(X) \times F(Y) \rightarrow G(X \wedge Y)$ by setting $m(f, g)=f \wedge g$, and 3.4 is applicable to this case.

(b) According to $1.10(\mathrm{e})$, the category weight $\operatorname{cwgt} \xi$ of a fibration $\xi$ is defined. It turns out that, for any fibrations $\xi, \eta$ over the same base $X$,

$$
\operatorname{cwgt}\left(\xi *_{X} \eta\right) \geq \operatorname{cwgt} \xi+\operatorname{cwgt} \eta \text {. }
$$

This can be deduced from 3.4, but we indicate a direct proof. Let $A, K, L$ be as in the proof of 3.4 , and let $s_{1}$ (resp. $s_{2}$ ) be a section of $\xi$ over $K$ (resp of $\eta$ over $L$ ). Take a function $\varphi: A \rightarrow[0,1]$ such that $\varphi^{-1}(0,1] \in K$ and $\varphi^{-1}[0,1) \in L$. We set

$$
s(x):=\left\langle s_{1}(x), \varphi(x), s_{2}(x)\right\rangle \in\{\text { the total space of } \xi * \eta\}, x \in A .
$$

Then $s$ is a desired section over $A$. Now, we can formulate (and, probably, exploit) the obvious analog of (3.7). 
The following result shows how Massey products help to estimate cat $X$. Let $H^{*}(-; R)$ denote singular cohomology with coefficients in a commutative ring $R$. Given a matrix $V$ over $H^{*}(X ; R)$, we set cwgt $V=\min \{\operatorname{cwgt} v\}$ where $v$ runs over all entries of $V$.

3.10. Theorem $([\mathrm{R} 2])$. Given $X \in \mathscr{T}$, let $V_{1}, \ldots, V_{n}$ be matrices over $H^{*}(X ; R)$. Suppose that the matrix Massey product $\left\langle V_{1}, \ldots, V_{n}\right\rangle$ is defined. If $0 \notin\left\langle V_{1}, \ldots, V_{n}\right\rangle$ then

$$
\text { cat } X \geq \min _{i}\left\{\operatorname{cwgt} V_{2 i}\right\}+\min _{i}\left\{\operatorname{cwgt} V_{2 i+1}\right\} \text {. }
$$

My feeling is that this result is somehow related to (3.7), but I can't say how explicitly.

4. Elements of high category weight. The results of the previous section show that it makes sense to search for (indecomposable) elements of high category weight. The first example of this kind was found by Fadell-Husseini [FH]. Namely, they proved that, for every odd prime $p$ and connected $X$,

$$
\operatorname{cwgt} \beta P^{n} u \geq 2 \text { if } u \in H^{2 n+1}(X ; \mathbb{Z} / p) .
$$

(Actually, they proved that $\operatorname{swgt} \beta P^{n} u \geq 2$.)

4.2. Theorem. Let $X \in \mathscr{T}$, and let $V_{1}, \ldots, V_{n}$ be matrices over $H^{*}(X ; R)$ (singular cohomology with coefficients in a commutative ring). Suppose that the matrix Massey product $\left\langle V_{1}, \ldots, V_{n}\right\rangle$ is defined. Then, for every $V \in\left\langle V_{1}, \ldots, V_{n}\right\rangle$ and every entry $u$ of $V$, we have cwgt $u \geq 2$.

This theorem was explicitly formulated in [R2]. Actually, it follows from 1.6, 1.8, and the result of Gugenheim-May [GM] that $\operatorname{Ker}\left\{\varepsilon^{*}: H^{*}(X) \rightarrow H^{*}(S \Omega X)\right\}$ contains all the matrix Massey products. Note that 4.2 implies (4.1) since $\beta P^{n} u \in\langle u, \ldots, u\rangle$ ( $p$ times) for every $u \in H^{2 n+1}(X ; \mathbb{Z} / p)$, Kraines $[\mathrm{K}]$.

Let $E, F$ be two spectra, and let $\theta: E \rightarrow F$ be a (stable) cohomology operation. Without loss of generality we can assume that $E=\left\{E_{n}\right\}$ and $F=\left\{F_{n}\right\}$ are $\Omega$-spectra, i.e., that there are weak homotopy equivalences $\sigma_{n}: E_{n} \rightarrow \Omega E_{n+1}$, etc. Then $\theta$ yields a family $\theta_{n}: E_{n} \rightarrow F_{n}$ of maps such that the diagram

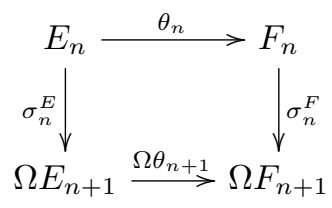

commutes up to homotopy. Following Strom [S2], set

$$
d=d(\theta)=\inf \left\{k \mid \theta_{k} \text { is an essential map }\right\}
$$

4.3. TheOREM ([S2]). $\operatorname{swgt} \theta_{n}=1$ for $n>d$, and $\operatorname{swgt} \theta_{d} \geq 2$.

Notice that $d(\theta)=2 n+1$ for $\theta=\beta P^{n}$. This gives us another proof of (4.1). Indeed, if $u \in H^{2 n+1}(X ; \mathbb{Z} / p)$ then

$$
\operatorname{swgt} \beta P^{n}(u)=\operatorname{swgt} \theta_{2 n+1}(u) \geq \operatorname{swgt}\left(\theta_{2 n+1}\right) \operatorname{swgt} u \geq 2 \operatorname{swgt} u \geq 2 .
$$

4.4. THEOREM ([S3]). Let $G$ be a discrete group, and let $E$ be a spectrum such that $\pi_{i}(E)=0$ for $i<m$. Then $\operatorname{swgt} u \geq k+m$ for every $u \in E^{k}(B G)$. 
For $k=2$ this result goes back to Fadell-Husseini [FH]. Notice that, by 2.6, swgt $u=k$ for every $u \in H^{k}(B G), u \neq 0$. As an application of 4.4, we note the following corollary.

4.5. Corollary. Let $M$ be a smooth manifold such that the structure group of its tangent bundle reduces to a discrete group $G$. If $w_{I}(M) \neq 0$ then cat $M \geq|I|$. Here $I=\left\{i_{1}, \ldots, i_{k}\right\}, w_{I}$ is the Stiefel-Whitney class $w_{i_{1}} \cdots w_{i_{k}}$ and $|I|=\sum i_{k}$.

Proof. It suffices to prove that swgt $w_{I} \geq|I|$. But this follows from 4.3 and 2.2(iii), since $w_{I}$ is induced from $B G$.

The last example of elements of high category weight is as follows.

4.6. TheOrem. Let $X$ be a simply connected rational space, and let $(\Lambda, d)$ be the minimal Sullivan model for $X$. If a cohomology class $x \in H^{*}(X ; \mathbb{Q})=H^{*}(\Lambda, d)$ has the form $x=\left[a_{1} \cdots a_{k}\right]$ for some $a_{i} \in \Lambda, \operatorname{dim} a_{i} \geq 0$, then $\operatorname{swgt} x \geq k$.

Notice that the $a_{i}$ are not assumed to be cocycles and, hence, we can't apply (3.6).

Pr o of. This follows from the result of Felix-Halperin $[\mathrm{FeH}, \S 3]$ that $p_{k}^{*}: H^{*}(X ; \mathbb{Q}) \rightarrow$ $H^{*}\left(P_{k}(X) ; \mathbb{Q}\right)$ annihilates all elements of the form $\left[a_{1} \cdots a_{k}\right]$.

\section{Detecting elements}

5.1. Definition. Let $F$ be as 1.9. An element $u \in F^{*}(X)$ is called a detecting element for $X$ if swgt $u=\operatorname{cat} X$.

We formulate the above definition for the general situation, but really we will apply it to the case when $F$ is a cohomology theory, as in $1.9(\mathrm{~d})$.

5.2. Theorem ([R2]). Let $X, Y$ be two connected pointed $C W$-spaces and let $E, G$ be two spectra. Suppose that there are detecting elements $u \in E^{*}(X), v \in G^{*}(Y)$. If $0 \neq u \otimes v \in(E \wedge G)^{*}(X \times Y)$ then $\operatorname{cat}(X \times Y)=$ cat $X+$ cat $Y$, and $u \bigotimes v$ is a detecting element for $X \times Y$.

Proof. By 2.2(i), 3.5 and 1.4,

$\operatorname{cat}(X \times Y) \geq \operatorname{swgt}(u \otimes v) \geq \operatorname{swgt} u+\operatorname{swgt} v=\operatorname{cat} X+\operatorname{cat} Y \geq \operatorname{cat}(X \times Y)$.

5.3. Corollary. If a pointed $C W$-space $X$ possesses a detecting element then cat $(X \times$ $\left.S^{n}\right)=\operatorname{cat} X+1$ for every $n \geq 0$. In other words, the Ganea conjecture holds for $X$.

Proof. We take a detecting element $u \in E^{*}(X)$ and apply 5.2 to the case $Y=$ $S^{n}, G^{n}(Y)=\Pi^{n}\left(S^{n}\right)$, and $v$ is given by the identity map $S^{n} \rightarrow S^{n}$. One can prove that $u \bigotimes v \neq 0$ (see e.g. [R2]), and the result follows.

For example, Strom [S1] proved that a $(q-1)$-connected $C W$-space $X$ possesses a detecting element if cat $X=[\operatorname{dim} X / q]$, and so such an $X$ satisfies the Ganea conjecture.

5.4. TheOREM ([R2]). Let $R$ be a ring spectrum, and let $E$ be an arbitrary $R$-module spectrum. Let $M^{n}, N^{n}$ be two closed connected $H \mathbb{Z}$-orientable PL manifolds, and let $f$ : $N \rightarrow M$ be a map of degree \pm 1 and such that $N$ is $R$-orientable. If $M$ possesses a detecting element $u \in E^{*}(M)$ then cat $f=$ cat $M$. In particular, cat $N \geq \operatorname{cat} M$.

Proof. It is easy to see that $f^{*}: E^{*}(M) \rightarrow E^{*}(N)$ is monic. So, $f^{*} u \neq 0$, and hence, by $2.2(\mathrm{i})$, cat $f \geq \operatorname{swgt} u=\operatorname{cat} M$. Hence, by 1.3 , cat $f=$ cat $M$. 
The results above show that it is useful and important to know whether a space possesses detecting elements. Consider the Puppe sequence

$$
P_{m}(X) \stackrel{p_{m}}{\rightarrow} X \stackrel{j_{m}}{\rightarrow} C_{m}(X):=C\left(p_{m}\right)
$$

where $p_{m}: P_{m}(X) \rightarrow X$ is the fibration (1.5) and $C\left(p_{m}\right)$ is the cone of $p_{m}$.

5.5. TheOrem ([R2]). Let $X$ be a pointed connected $C W$-space with cat $X=k<\infty$. If $j_{k}$ is stably essential (i.e., the stable homotopy class of $j_{k}$ is non-zero) then $X$ possesses a detecting element.

In fact, the stable homotopy class of $j_{m}$ can be treated as a universal (among cohomology functors) detecting element. Also, we note that, for every $X$ with cat $X=k$, the fibration $p_{k}: P_{k}(X) \rightarrow X$ is a detecting element (see 1.10(e)). However, unfortunately, it is difficult to apply analogs of $5.2-5.4$ to this case.

6. Manifolds. Given a PL manifold $M$, we denote by $\nu_{M}$ the stable normal bundle of $M$.

6.1. Theorem ([R2]). Let $M^{n}, n=\operatorname{dim} M \geq 4$, be a closed $(q-1)$-connected $P L$ manifold, $q \geq 1$. Suppose that there is a natural number $m$ such that $\nu_{M} \mid M^{(m)}$ is trivial and

$$
n \leq \min \{2 q \text { cat } M-4, m+q \text { cat } M-1\} .
$$

Then $M$ possesses a detecting element.

Putting $m=n+1$ and $m=1$, we get the following corollary.

6.2. Corollary. (i) Let $M$ be a closed $(q-1)$-connected stably parallelizable $P L$ manifold, $q \geq 1$. Suppose that $4 \leq \operatorname{dim} M \leq 2(q$ cat $M-2)$. Then $M$ possesses a detecting element.

(ii) Let $q \geq 1$, and let $M^{n}, n=\operatorname{dim} M \geq 4$ be a closed orientable $(q-1)$-connected $P L$ manifold such that $q$ cat $M=n$. Then $M$ possesses a detecting element. Moreover, there exists a detecting element $u \in H^{n}\left(M ; \pi_{n}\left(C_{n}(M)\right)\right)$.

6.3. Corollary. Let $M$ be as in 6.1. Then $\operatorname{cat}\left(M \times S^{m_{1}} \times \cdots \times S^{m_{n}}\right)=\operatorname{cat} M+n$ for any natural numbers $m_{1}, \ldots, m_{n}$.

Other results about the Ganea conjecture for manifolds can be found in [R1], [R2], [S1]. Based on 6.2 and 5.4, we get the following theorem.

6.4. Theorem. (i) Let $M^{n}$ be as in 6.2(i), and let $f: N^{n} \rightarrow M^{n}$ be a map of degree \pm 1 where $N$ is a stably parallelizable $P L$ manifold. Then cat $f=$ cat $M$. In particular, cat $N \geq \operatorname{cat} M$.

(ii) Let $f: N \rightarrow M$ be a map of degree \pm 1 of closed $H \mathbb{Z}$-orientable PL manifolds. If cat $M=\operatorname{dim} M$ then cat $f=\operatorname{cat} M=\operatorname{cat} N$.

6.5. Corollary. Let $M$ be an oriented $P L$ manifold with cat $M=\operatorname{dim} M$, and let $f: X \rightarrow M$ be a map of an arbitrary topological space such that

$$
f^{*}: H^{n}\left(M ; \pi_{n}\left(C_{n}(M)\right)\right) \rightarrow H^{n}\left(M ; \pi_{n}\left(C_{n}(M)\right)\right)
$$

is a monomorphism. Then cat $f=\operatorname{cat} M$. 
Proof. By 6.2, there is a detecting element $u \in H^{n}\left(M ; \pi_{n}\left(C_{n}(M)\right)\right)$, and $f^{*} u \neq 0$. Thus,

$$
\text { cat } f \geq \operatorname{swgt} u=\operatorname{cat} M \text {. }
$$

But, by $1.3(\mathrm{i})$, cat $f \leq$ cat $M$.

7. Applications to the Arnold conjecture. In [A, Appendix 9] Arnold proposed a beautiful conjecture concerning the relation between the number of fixed points of certain self-diffeomorphisms of a closed symplectic manifold $(M, \omega)$ and the minimum number of critical points of any smooth $\left(=C^{\infty}\right)$ function on $M$.

Let $\left(M^{2 n}, \omega\right)$ be a closed symplectic manifold. A symplectomorphism $\phi: M \rightarrow M$ (i.e., a diffeomorphism with $\phi^{*} \omega=\omega$ ) is called Hamiltonian (or exact) if it belongs to the flow of a time-dependent Hamiltonian vector field on $M$. See [HZ] or [MS] for details. We define $\operatorname{Arn}(M, \omega)$ to be the minimum number of fixed points for any Hamiltonian symplectomorphism of $M$. The Arnold conjecture claims that the following inequality holds for every closed symplectic manifold $(M, \omega)$ :

$$
\operatorname{Arn}(M, \omega) \geq \operatorname{Crit} M \text {. }
$$

The conjecture, usually (but not universally) weakened by replacing Crit $M$ by the cuplength of $M$, has been proved under various hypotheses for various classes of manifolds ([CZ], $[\mathrm{H}],[\mathrm{Fl1}],[\mathrm{Fl}]$ ). Here the following theorem (formulated explicitly in [R2] and based on Floer's approach) plays the crucial role.

7.1. TheOREM. Let $(M, \omega)$ be a closed connected symplectic manifold such that $\left.\omega\right|_{\pi_{2}(M)}$ $=0=\left.c_{1}(M)\right|_{\pi_{2}(M)}$. Then there exists a map $f: X \rightarrow M$ with the following properties:

(i) $X$ is a compact metric space;

(ii) $1+$ cat $f \leq \operatorname{Arn}(M, \omega)$;

(iii) The homomorphism $f^{*}: H^{n}(M ; G) \rightarrow H^{n}(X ; G)$ is a monomorphism for every coefficient group $G$.

The following theorem is proved in [R3] and [RO].

7.2. TheOREM. Let $\left(M^{2 n}, \omega\right)$ be a closed connected symplectic manifold with

$$
\left.\omega\right|_{\pi_{2}(M)}=0=\left.c_{1}\right|_{\pi_{2}(M)} .
$$

Then $\operatorname{Arn}(M, \omega) \geq$ Crit $M$, i.e., the Arnold conjecture holds for $M$.

Pro of. First, note that, by 3.8 , cat $M=2 n=\operatorname{dim} M$. Hence,

$$
1+\operatorname{cat} M \leq \operatorname{Crit} M \leq 1+\operatorname{dim} M=1+\operatorname{cat} M
$$

(the last inequality is a theorem of Takens $[\mathrm{T}]$ ). So, $1+$ cat $M=$ Crit $M$. Thus, in view of 7.1 , it remains to prove that cat $f \geq$ cat $M$ where $f$ is the map from 7.1. We give two proofs of this inequality.

First proof. This follows from 6.5.

Second proof. Rudyak-Oprea [RO] proved that swgt $\omega=2$. Thus, since $f^{*} \omega^{n} \neq 0$,

$$
\text { cat } f \geq \operatorname{swgt} \omega^{n} \geq 2 n=\operatorname{cat} M \text {. }
$$




\section{References}

[A] V. I. Arnold, Mathematical Methods in Classical Mechanics, Springer, Berlin 1989.

[B] A. Bassi, Su alcuni nuovi invarianti delle varietà topologiche, Annali di Math. 16 (1935), 275-297.

[BG] I. Berstein and T. Ganea, The category of a map and of a cohomology class, Fund. Math. 50 (1961/2), 265-279.

[CZ] C. Conley and E. Zehnder, The Birkhoff-Lewis Fixed Point Theorem and a Conjecture of V.I. Arnold, Invent. Math. 73 (1983), 33-49.

[FH] E. Fadell and S. Husseini, Category weight and Steenrod operations, Boletin de la Sociedad Matemática Mexicana 37 (1992), 151-161.

[F] I. Fary, Sur la catégorie des classes de homologie d'un espace, Proc. Intern. Congr. Math. 1954, 2, 215, North-Holland, Amsterdam 1957.

[FeH] Y. Felix and S. Halperin, Rational L.-S. category and its applications, Trans. Amer. Math. Soc. 273 (1982), 1-38.

[Fet] A. I. Fet, A connection between the topological properties and the number of extremals on a manifold (Russian), Doklady AN SSSR 88 (1953), 415-417.

[Fl1] A. Floer, Cuplength estimates on Lagrangian intersections, Comm. Pure Appl. Math 42 (1989), 335-356.

[Fl2] A. Floer, Symplectic fixed points and holomorphic spheres, Commun. Math. Phys. 120 (1989), 575-611.

[Fox] R. Fox, On the Lusternik-Schnirelmann category, Ann. of Math. 42 (1941), 333-370.

[FE] S. Froloff and L. Elsholz, Limite inférieure pour le nombre des valeurs critiques d'une fonction, donné sur une variété, Math. Sbornik 42, 5 (1935), 637-643.

[G] T. Ganea, Some problems on numerical homotopy invariants, Symposium in Algebraic Topology, Seattle 1971, 23-30, Lecture Notes in Mathematics 249, Springer, Berlin 1971.

[GM] V. K. A. M. Gugenheim and J. P. May, On the Theory and Applications of Differential Torsion Products, Memoirs Amer. Math. Soc. 142, AMS, Providence, Rhode Island 1974.

[H] H. Hofer, Lusternik-Schnirelmann theory for Lagrangian intersections, Annales de l'inst. Henri Poincaré- analyse non linéaire 5 (1988), 465-499.

[HZ] H. Hofer and E. Zehnder, Symplectic Invariants and Hamiltonian Dynamics, Birkhäuser, Basel 1994.

[I] N. Iwase, Ganea's conjecture on Lusternik-Schnirelmann category, preprint 1997.

[J] I. M. James, On category, in the sense of Lusternik-Schnirelmann, Topology 17 (1978), 331-349.

[K] D. Kraines, Massey higher products, Trans. Amer. Math. Soc. 124 (1966), 431-449.

[LS] L. A. Lusternik and L. G. Schnirelmann, Méthodes topologiques dans les problèmes variationnels, Hermann, Paris 1934.

[MS] D. McDuff and D. Salamon, Introduction to Symplectic Topology, Clarendon Press, Oxford 1995.

[R1] Yu. B. Rudyak, On the Ganea conjecture for manifolds, Proc. Amer. Math. Soc. 125 (1997), 2511-2512.

[R2] Yu. B. Rudyak, On category weight and its applications, Topology 38 (1999), 37-55.

[R3] Yu. B. Rudyak, On analytical applications of stable homotopy (the Arnold conjecture, critical points), Math. Zeitschrift, to appear.

[RO] Yu. B. Rudyak and J. Oprea, On the Lusternik-Schnirelmann Category of Symplectic Manifolds and the Arnold Conjecture, Math. Zeitschrift, to appear. 
[S1] J. Strom, Two Special Cases of Ganea's Conjecture, Trans. Amer. Math. Soc., to appear.

[S2] J. Strom, Essential category weight, preprint, 1997.

[S3] J. Strom, Essential category weight and classifying spaces, preprint, 1997.

[Sv] A. Svarc, The genus of a fiber space, Amer. Math. Soc. Translations 55 (1966), 49-140.

[Sw] R. W. Switzer, Algebraic Topology - Homotopy and Homology, Springer, Berlin 1975.

[T] F. Takens, The minimal number of critical points of a function on a compact manifold and the Lusternik-Schnirelmann category, Invent. Math. 6 (1968), 197-244.

[To] G. Toomer, Lusternik-Schnirelmann Category and the Moore Spectral Sequence, Math. Z. 138 (1974), 123-143.

[W] J. H. C. Whitehead, Simplicial spaces, nuclei, and m-groups, Proc. London Math. Soc. 45 (1939), 243-327, 